\title{
On Polynomial-Time Learnability in the Limit of Strictly Deterministic Automata
}

\author{
TAKASHI YOKOMORI \\ yokomori@cs.uec.ac.jp \\ Department of Computer Science and Information Mathematics, University of Electro-Communications, 1-5-1 \\ Chofugaoka, Chofu, Tokyo, 182 Japan
}

Editor: Leonard Pitt

\begin{abstract}
This paper deals with the polynomial-time learnability of a language class in the limit from positive data, and discusses the learning problem of a subclass of deterministic finite automata (DFAs), called strictly deterministic automata (SDAs), in the framework of learning in the limit from positive data. We first discuss the difficulty of Pitt's definition in the framework of learning in the limit from positive data, by showing that any class of languages with an infinite descending chain property is not polynomial-time learnable in the limit from positive data. We then propose new definitions for polynomial-time learnability in the limit from positive data. We show in our new definitions that the class of SDAs is iteratively, consistently polynomial-time learnable in the limit from positive data. In particular, we present a learning algorithm that learns any SDA $M$ in the limit from positive data, satisfying the properties that $(i)$ the time for updating a conjecture is at most $\mathrm{O}(\ell m)$, (ii) the number of implicit prediction errors is at most $\mathrm{O}(\ell n)$, where $\ell$ is the maximum length of all positive data provided, $m$ is the alphabet size of $M$ and $n$ is the size of $M$, (iii) each conjecture is computed from only the previous conjecture and the current example, and (iv) at any stage the conjecture is consistent with the sample set seen so far. This is in marked contrast to the fact that the class of DFAs is neither learnable in the limit from positive data nor polynomial-time learnable in the limit.
\end{abstract}

Keywords: polynomial-time learnability in the limit, iterative learning, strictly deterministic automata

\section{Introduction}

In the study of inductive learning of formal languages, Gold (1967) showed that any class of languages containing all finite sets and at least one infinite set (which is called a superfinite class) is not learnable in the limit from positive data (that is, from the examples in the target language). This fact was shocking in the sense that even the class of regular languages is not learnable in the limit from positive data. Angluin (1980) has given several conditions for a class of languages to be learnable in the limit from positive data, and presented some examples of learnable classes. She has also proposed subclasses of regular languages called $k$-reversible languages for each non-negative integer $k$ and shown these classes are learnable in the limit from positive data with a polynomialtime algorithm for updating conjectures (Angluin, 1982). Motivated by a question posed by Angluin, however, one natural question has been recently recognized as significant: In what sense should we analyse the time complexity of an "in-the-limit" algorithm ? Because there are a great variety of possible definitions for polynomial-time learnability 
in the limit. Among others, Pitt has proposed the following definition for polynomialtime learnability in the limit from positive and negative data (Pitt, 1989). Informally, a class of concepts (or representations) $\mathcal{M}$ is polynomial-time learnable in the limit if and only if there is an algorithm $\mathcal{A}$ which, given a sequence of strings accepted by $M$ in $\mathcal{M}$, learns an equivalent $M^{\prime}$ in $\mathcal{M}$ in the limit, with the property that there exist polynomials $p$ and $q$ such that the time for updating a conjecture is at most $p(n, N)$ and the number of times $\mathcal{A}$ makes a wrong conjecture is at most $q(n)$, where $n$ is the size of $M$, and $N$ is the sum of lengths of data provided. It turned out, however, that this definition was too strong to have positive results for non-trivial language classes. For example, a recent result shows that even the class of all finite languages or a subclass of regular languages called zero-reversible languages is not polynomial-time learnable in the limit (Angluin, 1990; Pitt, 1989). In fact, to the author's knowledge, no non-trivial class of languages has been yet proven polynomial-time learnable in the limit in this definition. It is beyond question that the polynomial-time learnability in the limit (in particular, from positive data) is of great significance from not only the theoretical but also the practical point of view.

In our previous paper (Tanida \& Yokomori, 1992), we have introduced a subclass of DFAs called strictly deterministic automata (SDAs) and shown that the class is learnable in the limit from positive data requiring polynomial time for updating conjectures. An SDA is intuitively a state transition graph in which the set of labels $W$ for edges is a finite subset of strings over an alphabet $\Sigma$. For each symbol $a$ in $\Sigma$ there is at most one $w$ in $W$ starting with $a$. The motivation for introducing the notion of an SDA is explained as follows. Consider the following sentences :

$$
\begin{aligned}
& \text { "you feel very happy" } \\
& \text { "she is very very happy" } \\
& \text { "she came long long ago" }
\end{aligned}
$$

These are recognized by, for instance, the acceptor $M$ in Figure 1. We observe that all edges of $M$ are labeled by words that all start with distinct letters, and that double occurrences of an identical word (say, "very" or "long") ensure the existence of a self-loop in the transition graph. These features lead to a language class of specific sentences with which we are concerned in the present paper. This notion is also motivated by the work on Szilard languages of linear grammars (Mäkinen, 1990). The class of Szilard languages of linear grammars is a proper subclass of the class of zero-reversible languages, while our class of languages properly includes the class of Szilard languages of linear grammars, but is incomparable to the class of zero-reversible languages.

In this article, we first discuss the difficulty of Pitt's definition in the framework of learning in the limit from positive data, by showing that any class of languages with an infinite descending chain property is not polynomial-time learnable in the limit from positive data. Since most of the existing language classes satisfy the property, this result strongly encourages us to propose new definitions for polynomial-time learnability in the 


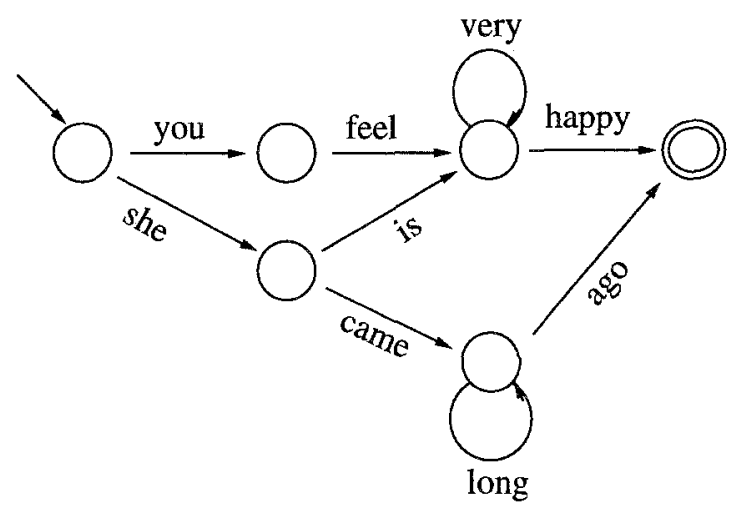

Figure 1. A Simple Natural Language Recognizer

limit from positive data. In fact, we propose two new definitions for polynomial-time learnability in the limit from positive data by making modifications to a straightforwardly restricted version of Pitt's definition for the case of positive data only.

Then, we make a visit to the class of SDAs again to generalize and strengthen the learnability results of SDAs in the new definition. We show that the class of SDAs is polynomial-time learnable in the limit from positive data, by presenting an improved version of the learning algorithm given in Tanida and Yokomori (1992). This result is in marked contrast to the fact that the class of DFAs is neither learnable in the limit from positive data nor polynomial-time learnable in the limit. As corollaries, it follows that the class of SDAs is learnable in polynomial time via equivalence queries only and it is also PAC-learnable in polynomial time from positive examples only. The main results in this paper extend and supplement those in not only Tanida and Yokomori (1992) but also Mäkinen (1990). Further, we discuss an interesting feature of our learning algorithm, namely, iterative consistency which enables the algorithm to be efficient in space as well.

\section{Definitions}

\subsection{Basic Definitions and Notations}

We assume the reader to be familiar with the basic notions in automata and formal language theory. For the notions and notations not stated here, see, e.g., Hopcroft and Ullman (1979). An alphabet $\Sigma$ is a finite set of symbols. For any finite set $S$ of finitelength strings over $\Sigma$, we denote by $S^{*}$ (respectively, $S^{+}$) the set of all finite-length strings obtained by concatenating zero (one, resp.) or more elements of $S$, where the concatenation of strings $u$ and $v$ is simply denoted by $u v$. In particular, $\Sigma^{*}$ denotes the set of all finite-length strings over $\Sigma$. The string of length 0 (the empty string) is denoted 
by $\lambda$. By len $(w)$ we denote the length of a string $w$. A language over $\Sigma$ is any subset $L$ of $\Sigma^{*}$. The cardinality of a set $S$ is denoted by $|S|$. In a directed graph, called a transition graph, an edge from node $p$ to node $q$ labeled $u$ is denoted by $(p, u, q)$ and is called a transition. A string $u\left(\in \Sigma^{+}\right)$is called the label of $(p, u, q)$. For a language $L \subseteq \Sigma^{*}$ and $x \in \Sigma^{*}$, let $x \backslash L=\{y \mid x y \in L\}$.

\subsection{Polynomial-time Learning in the Limit from Positive Data - Difficulty and New Definitions}

Let $\Sigma$ be fixed and let $\mathcal{M}$ be the class of acceptors to be learned, where each $M \in \mathcal{M}$ specifies a language $L$ over $\Sigma$, denoted by $L=L(M)$. Let $L$ be a language such that $L=L(M)$ for some $M$ in $\mathcal{M}$. A string in $L$ is called a positive example of $L$ and positive examples are often called positive data. A positive presentation of the language $L$ is any infinite sequence of strings such that every string $w \in L$ occurs at least once in the sequence, and no other strings occur in the sequence. In our model of learning, a learning algorithm $\mathcal{A}$ takes, as an input, an arbitrary positive presentation of $L$ : $w_{1}, w_{2}, \cdots$, and outputs an infinite sequence of acceptors (conjectures): $M_{1}, M_{2}, \cdots$. An algorithm $\mathcal{A}$ is said to learn an acceptor $M$ in the limit from positive data if and only if for any positive presentation of $L(M)$ the infinite sequence of acceptors in $\mathcal{M}$ produced by $\mathcal{A}$ satisfies the property that there exists $M^{\prime}$ in $\mathcal{M}$ such that for all sufficiently large $i$, the $i$-th conjecture (acceptor) $M_{i}$ is identical to $M^{\prime}$ and $L\left(M^{\prime}\right)=L(M)$. A class of acceptors $\mathcal{M}$ is learnable in the limit from positive data if and only if there exists an algorithm $\mathcal{A}$ that, for any $M$ in $\mathcal{M}$, learns $M$ in the limit from positive data. For each $i \geq 1$, let $w_{i}$ be the $i$-th example provided and $M_{i}$ be the $i$-th conjectured acceptor produced by an algorithm $\mathcal{A}$. Then, $\mathcal{A}$ is said to make an implicit error of prediction at the $i$-th step if and only if the conjecture $M_{i}$ fails to accept the $(i+1)$-st example $w_{i+1}$.

From the original Pitt's definition (Pitt, 1989) for polynomial-time learnability in the limit, one may propose the following definition for the case of positive data only.

Definition $1 \mathrm{~A}$ class $\mathcal{M}$ is polynomial-time learnable in the limit from positive data if and only if (1) $\mathcal{M}$ is learnable in the limit from positive data, and (2) the learning algorithm $\mathcal{A}$ in (1) satisfies the property that there exist polynomials $p, q$ such that for any $n$, for any $M$ of size $n$, and for any positive presentation of $L(M)$, the time used by $\mathcal{A}$ between receiving the $i$-th example $w_{i}$ and outputting the $i$-th conjectured acceptor $M_{i}$ is at most $p\left(n, l_{1}+\cdots+l_{i}\right)$, and the number of implicit errors of prediction made by $\mathcal{A}$ is at most $q(n)$, where $l_{j}=\operatorname{len}\left(w_{j}\right)(j=1,2, \ldots, i)$.

However, no non-trivial class of acceptors (or languages) has been yet proven polynomia time learnable in the limit in this definition. (In fact, it has been proved that even the class of all DFAs recognizing only finite languages is not polynomial-time learnable in the limit (Angluin, 1990). On the other hand, the class of DFAs is polynomial-time learnable in the limit with the help of membership queries (Angluin, 1987).) 
Moreover, we can show the following negative result in this definition.

THEOREM 1 Let $\mathcal{M}$ be any class of acceptors with the property that there exists an infinite sequence of acceptors $M_{0}, M_{1}, M_{2}, \cdots$ such that $L\left(M_{0}\right) \supset L\left(M_{1}\right) \supset L\left(M_{2}\right) \cdots$, that is, the sequence forms an infinite descending chain with respect to the inclusion relation. Then, $\mathcal{M}$ cannot be learned according to Definition 1.

Proof: Suppose that there exists an infinite descending chain : $L\left(M_{0}\right), L\left(M_{1}\right)$, $L\left(M_{2}\right), \cdots$, where for each $i \geq 0 L\left(M_{i}\right)$ properly contains $L\left(M_{i+1}\right)$. Let $\mathcal{A}$ be any algorithm that learns every $M$ in $\mathcal{M}$ in the limit from positive data. We show that for every positive integer $k$, we can construct a positive presentation of $L\left(M_{0}\right)$ on which $\mathcal{A}$ must make at least $k$ implicit errors of prediction.

Begin by enumerating the elements of $L\left(M_{k}\right)$ and providing them as input to $\mathcal{A}$ until the output sequence of conjectures of $\mathcal{A}$ converges to an acceptor equivalent to $M_{k}$. This must happen in finite time, since $\mathcal{A}$ learns every $M$ in $\mathcal{M}$ in the limit from positive data. When this happens, choose any element of $L\left(M_{k-1}\right)-L\left(M_{k}\right)$ as the next example in the positive presentation. On this element, $\mathcal{A}$ makes an implicit error of prediction. Then, we continue the construction of positive presentation by enumerating the elements of $L\left(M_{k-1}\right)$ until the output sequence of $\mathcal{A}$ converges to an acceptor equivalent to $M_{k-1}$. At this point, choose any element of $L\left(M_{k-2}\right)-L\left(M_{k-1}\right)$ as the next example in the input sequence on which $\mathcal{A}$ makes an implicit error of prediction, and we continue with an enumeration of $L\left(M_{k-2}\right)$.

Thus, if we continue in this way, then the output sequence of $\mathcal{A}$ converges to an acceptor equivalent to $M_{0}$ after having made at least $k$ implicit errors of prediction.

Let $n_{0}$ be the size of $M_{0}$. Then, since $n_{0}$ is some fixed value, no function $f(n)$ has the property that $k \leq f\left(n_{0}\right)$ for all positive integers $k$. Thus, no algorithm that learns every $M$ in $\mathcal{M}$ in the limit from positive data can make a number of implicit errors of prediction bounded by a polynomial in the size of the target acceptor.

Since most of the non-trivial classes of acceptors have the infinite descending chain property stated in the theorem, this implies that Definition 1, a straightforward modification of Pitt's definition, is too restrictive to obtain any positive result of polynomial-time learnability in the limit from positive data, leading us to new definitions.

We now propose the following two new definitions for polynomial-time learnability in the limit from positive data.

Definition 2 A class $\mathcal{M}$ is polynomial-time learnable in the limit from positive data if and only if (1) $\mathcal{M}$ is learnable in the limit from positive data, and (2) the learning algorithm $\mathcal{A}$ in (1) satisfies the property that there exist polynomials $p, q$ such that for any $n$, for any $M$ of size $n$, and for any positive presentation of $L(M)$, the time used by $\mathcal{A}$ between receiving the $i$-th example $w_{i}$ and outputting the $i$-th conjectured acceptor $M_{i}$ is at most $p\left(n, l_{1}+\cdots+l_{i}\right)$, and the number of implicit errors of prediction made 
by $\mathcal{A}$ is at most $q(n, \ell)$, where $l_{j}=\operatorname{len}\left(w_{j}\right)(j=1,2, \ldots, i), \quad \ell$ is the maximum length of any positive example provided up to that point of the run of the learning algorithm, and the size of $M$ is assumed to be appropriately defined.

Definition 3 A class $\mathcal{M}$ is polynomial-time learnable in the limit from positive data in the strict sense if and only if, in addition to all the conditions in Definition 2, the algorithm $\mathcal{A}$ also satisfies the property that for any target acceptor $M$ in $\mathcal{M}$ and for any positive presentation of $L(M)$, each conjectured acceptor $M_{i}$ from $\mathcal{A}$ accepts a subset of $L(M)$, i.e., it holds that for all $i \geq 1, L\left(M_{i}\right) \subseteq L(M)$ (the subset requirement).

In comparison to Pitt's definition for polynomial-time learnability in the limit (Pitt, 1989), there are two major differences among these definitions. That is, in the new definitions above, the algorithm is allowed to have the maximum length of all data supplied as a new parameter for a polynomial that bounds the number of implicit errors of prediction (in (2)), while Definition 3, in turn, requires the algorithm to confine its conjectures to subsets of the target languages. Note that the learnability in Definition 3 obviously implies the learnability in Definition 2.

In the next section, we will introduce a non-trivial subclass of DFAs and show the class is polynomial-time learnable in the limit from positive data in the sense of Definition 2. In fact, we prove even a stronger result in the sense of Definition 3 whose introduction is motivated by the fact that it has some implications to other types of learning paradigms, in particular, learning with equivalence queries (Angluin, 1987) and PAC-Learning (Valiant, 1984)(see Corollary 17).

\subsection{Strictly Deterministic Automata and Their Languages}

We introduced in Tanida and Yokomori (1992) the notion of strictly deterministic automata which was defined in two steps, that is, first by extending the notion of (usual) deterministic finite automata and then by making a certain restriction on them. In this article, we will reformalize the notion in an extended manner.

Definition 4 (Wood, 1987) An extended deterministic finite automaton (EDFA) $M$ over $\Sigma$ is a 5 -tuple $\left(Q, T, \delta, p_{0}, F\right)$, where

$Q$ is a finite, nonempty set of states,

$T\left(\subseteq \Sigma^{+}\right)$is a finite set of strings,

$\delta: Q \times T \rightarrow Q$ is a state transition function (possibly) partially defined,

$p_{0}$ is the initial state in $Q$, and

$F(\subseteq Q)$ is a set of final states. 


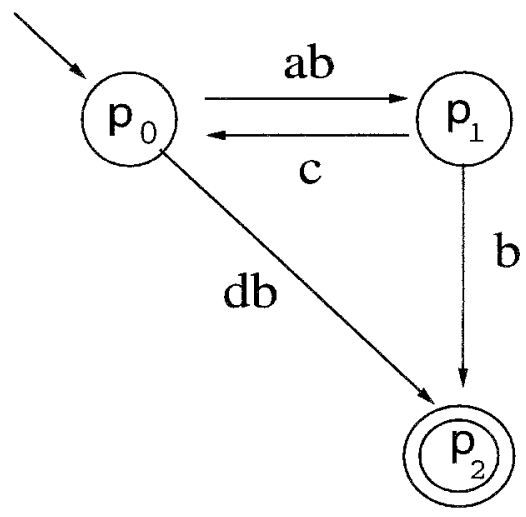

Figure 2. An SDA $M$ accpeting $L(M)$

For convenience, we sometimes take $\delta$ as the set of transitions $\{(p, u, q) \mid \delta(p, u)=q\}$.

Definition 5 [Strictly Deterministic Automaton] Let $M=\left(Q, T, \delta, p_{0}, F\right)$ be an EDFA. Then, $M$ is a strictly deterministic automaton (SDA) if and only if $M$ satisfies the following:

(1) for any $u \in T$, there uniquely exists a pair $(p, q)$ such that $(p, u, q) \in \delta$ (i.e., the uniqueness of labels).

(2) for any $u_{1}, u_{2} \in T$, the first symbol of $u_{1}$ differs from that of $u_{2}$. (We say that $T$ has the strict prefix property.)

A partial function $\delta$ is often extended from $Q \times T$ to $Q \times T^{*}$ in a usual manner: for all $p \in Q, x \in T^{*}, u \in T$

$$
\begin{aligned}
& \delta(p, \lambda)=p \\
& \delta(p, x u)=\delta(\delta(p, x), u) .
\end{aligned}
$$

The language accepted by $M$, denoted by $L(M)$, is defined by $\left\{w \in \Sigma^{*} \mid \delta\left(p_{0}, w\right) \in F\right\}$. A language $L$ is called a strictly regular language (SRL) if $L=L(M)$ for some SDA $M$.

Note. Besides (1) and (2) above, the original definition of an SDA in Tanida and Yokomori (1992) requires an additional condition which disallows multi-edges in the transition graph of $M$.

Example 1 Let $\Sigma=\{a, b, c, d\}$. Consider an EDFA $M=\left(\left\{p_{0}, p_{1}, p_{2}\right\},\{a b, b, c, d b\}\right.$, $\left.\delta, p_{0},\left\{p_{2}\right\}\right)$, where $\delta=\left\{\left(p_{0}, a b, p_{1}\right),\left(p_{0}, d b, p_{2}\right),\left(p_{1}, c, p_{0}\right),\left(p_{1}, b, p_{2}\right)\right\}$. Then, $M$ is an SDA and $L(M)$ is the language expressed by the regular expression $(a b c)^{*}(a b b+d b)$. The transition graph of $M$ is shown in Figure 2. 
In what follows, we fix the alphabet $\Sigma$ for SDAs or SRLs. Further, we assume that each SDA $M=\left(Q, T, \delta, p_{0}, F\right)$ has no useless states, where a state $q \in Q$ is said to be useless if it is not on any path from the initial state $p_{0}$ to a final state in $F$.

\subsection{Characterization Results on SDAs}

The next result is basic for all characterization results we will obtain in the sequel.

LEMMA 2 Let $M=\left(Q, T, \delta, p_{0}, F\right)$ be an $S D A$, and $p, p^{\prime}, q, q^{\prime}$ be in $Q$, and $\alpha \in T^{+}$. If $\delta(p, \alpha)=q$ and $\delta\left(p^{\prime}, \alpha\right)=q^{\prime}$, then $p=p^{\prime}$ and $q=q^{\prime}$.

Proof: Let $\alpha=u_{1} \cdots u_{n} ;\left(u_{i} \in T, 1 \leq i \leq n\right)$. We will show by induction on $n$ that if $\delta(p, \alpha)=q$ and $\delta\left(p^{\prime}, \alpha\right)=q^{\prime}$, then $p=p^{\prime}$ and $q=q^{\prime}$. Assume that $n=1$, then clearly the claim holds from the definition of SDA. Let us assume the claim holds for $n=k(k \geq 1)$. Let $\beta=\alpha u_{k+1}$, where $\alpha=u_{1} \cdots u_{k}$, and $u_{k+1} \in T$. Then,

$$
\begin{aligned}
\delta(p, \beta) & =\delta\left(p, u_{1} \cdots u_{k} u_{k+1}\right) \\
& =\delta\left(\delta(p, \alpha), u_{k+1}\right) \\
& =\delta\left(r, u_{k+1}\right), \quad \text { where } r=\delta(p, \alpha) \\
& =q .
\end{aligned}
$$

Similarly, we have that $\delta\left(p^{\prime}, \beta\right)=\delta\left(r^{\prime}, u_{k+1}\right)=q^{\prime}$, where $r^{\prime}=\delta\left(p^{\prime}, \alpha\right)$. Then, from the inductive hypothesis, $p=p^{\prime}$ and $r=r^{\prime}$, and hence $q=q^{\prime}$. Thus, the claim holds for $k=n+1$, which completes the proof.

From Lemma 2, we have the following corollary.

Corollary 3 Let $M=\left(Q, T, \delta, p_{0}, F\right)$ be an $S D A$, and let $u_{1}, u_{2}$ be in $T^{*}$ and $v$ be in $T^{+}$. If $M$ accepts $u_{1} v$ and $u_{2} v$, then $\delta\left(p_{0}, u_{1}\right)=\delta\left(p_{0}, u_{2}\right)$.

A regular language $L$ is zero-reversible if and only if for $u_{1}, u_{2} \in \Sigma^{*}$ and $v \in \Sigma^{+}$, whenever $u_{1} v, u_{2} v$ are in $L$, it holds that $u_{1} \backslash L=u_{2} \backslash L$ (Angluin, 1982). Corollary 3 means that an SRL is zero-reversible if it is restricted to $T^{*}$.

Example 2 Consider the strictly regular language $L=L(M)$ in Example 1. Clearly, $L$ is not zero-reversible, because, for example, $a b b$ and $d b$ are both in $L$ but $a b \backslash L \neq d \backslash L$. On the other hand, the language of the regular expression $a^{*} b a^{*}$ is zero-reversible, but not strictly regular, which can be proved using Lemma 2 .

Since there is a language which is both strictly regular and zero-reversible (e.g., the language of the regular expression $a^{*} b$ ), the following theorem holds. 
THEOREM 4 The class of strictly regular languages is incomparable to but not disjoint with the class of zero-reversible languages.

Using Lemma 2, it is easy to obtain the next lemma which gives an interesting property of SRLs.

LEMMA 5 Let $M=\left(Q, T, \delta, p_{0}, F\right)$ be an arbitrary SDA. Then, for $x, z \in \Sigma^{*}, y \in \Sigma^{+}$, if $x z, x y z$ and $x y y z$ are in $L(M)$, then for each $i \geq 0, x y^{i} z$ is in $L(M)$.

Proof: Let $v$ be the longest common prefix of $z$ and $y z$. Then, $x v$ is the longest common prefix of $x z$ and $x y z$, and $x y v$ is the longest common prefix of $x y z$ and $x y y z$. From the strict prefix property of an SDA, it is easy to see that if $M$ accepts two strings $x$ and $y$, then the longest common prefix of $x$ and $y$ reaches a state of $M$ from $q_{0}$. Hence, let $p_{1}=\delta\left(p_{0}, x v\right)$ and $p_{2}=\delta\left(p_{0}, x y v\right)$.

Since $v$ is the longest common prefix of $z$ and $y z$, it is also the longest common prefix of $z$ and $y v$. To see this, consider the following two cases. Case (1) $\operatorname{len}(v) \leq \operatorname{len}(y)$ : Then, $v$ is also clearly the longest common prefix of $z$ and $y v$. Case (2) $\operatorname{len}(v)>\operatorname{len}(y)$ : Let $z^{\prime}\left(\in \Sigma^{*}\right)$ and $z^{\prime \prime}\left(\in \Sigma^{+}\right)$be strings such that $z=v z^{\prime}$ and $v=y z^{\prime \prime}$. Then, $v$ can be also split as $(v=) z^{\prime \prime} v^{\prime}$ for some $v^{\prime} \in \Sigma^{*}$, where $z=z^{\prime \prime} v^{\prime} z^{\prime}$. Note that $z=v z^{\prime}=\left(y z^{\prime \prime}\right) z^{\prime}$, $y z=y\left(v z^{\prime}\right)=y\left(z^{\prime \prime} v^{\prime}\right)\left(z^{\prime}\right)=\left(y z^{\prime \prime}\right) v^{\prime} z^{\prime}$ and $y v=y\left(z^{\prime \prime} v^{\prime}\right)=\left(y z^{\prime \prime}\right) v^{\prime}$. Since $v\left(=y z^{\prime \prime}\right)$ is the longest common prefix of $z$ and $y z, v$ is also the longest common prefix of $z$ and $y v$. Thus, in either case, we conclude that $v$ is also the longest common prefix of $z$ and $y v$. This implies that there exists a string $y^{\prime}$ such that $y v=v y^{\prime}$. Since $y$ is non-empty, $y^{\prime}$ is non-empty. We now prove by induction on $j$ the following : [Claim] for each $j \geq 0, y^{j} v=v\left(y^{\prime}\right)^{j}$. The case when $j=0$ or $j=1$ is trivially true. Now consider

$$
\begin{aligned}
y^{j} v & =y y^{j-1} v \\
& =y v\left(y^{\prime}\right)^{j-1} \quad(\text { by inductive hypothesis) } \\
& \left.=v y^{\prime}\left(y^{\prime}\right)^{j-1} \quad \text { (since } y v=v y^{\prime}\right) \\
& =v\left(y^{\prime}\right)^{j} \quad \text { (End of the proof of Claim). }
\end{aligned}
$$

Let $z=v z^{\prime}$. Then, since $v$ is a prefix of $z$, it holds that

$$
x y z=x y v z^{\prime}=x v y^{\prime} z^{\prime} \text { and } x y y z=x y y v z^{\prime}=x y v y^{\prime} z^{\prime} .
$$

Thus, as we have seen above, since $p_{1}=\delta\left(p_{0}, x v\right)$ and $p_{2}=\delta\left(p_{0}, x y v\right)$, we have that $\delta\left(p_{1}, y^{\prime} z^{\prime}\right)=q_{f} \in F$ and $\delta\left(p_{2}, y^{\prime} z^{\prime}\right)=q_{f}^{\prime} \in F$. Since $y^{\prime} z^{\prime}$ is non-empty, from Lemma 2, we conclude that $p_{1}=p_{2}$ (and $q_{f}=q_{f}^{\prime}$ ). Since $x y v=x v y^{\prime}$, this implies that $\delta\left(p_{0}, x v\right)=p_{1}=p_{2}=\delta\left(p_{0}, x v y^{\prime}\right)$ and therefore, $p_{1}=\delta\left(p_{1}, y^{\prime}\right)$. Thus, for each $j \geq 0$, every string of the form $x v\left(y^{\prime}\right)^{j} z^{\prime}$ is accepted by $M$, while it holds that

$$
x v\left(y^{\prime}\right)^{j} z^{\prime}=x y^{j} v z^{\prime}=x y^{j} z .
$$

Therefore, for each $j \geq 0, M$ accepts every string of the form $x y^{j} z$. 


\section{Learning Strictly Deterministic Automata}

\subsection{The Learning Algorithm LA}

We present a learning algorithm $L A$ for SDAs which comprises four subprocedures named "UPDATE", "REFINE", "PARSE" and "CONSTRUCT"; we first give intuitive descriptions of these procedures.

Let $T$ be a set of strings corresponding to edge labels of a conjectured SDA to be constructed by $L A$. We say that $T$ can parse a string $w$ iff $w$ can be uniquely written as the concatenation of strings from the set $T$, i.e., if $w=x_{1} \cdots x_{m}=x_{1}^{\prime} \cdots x_{n}^{\prime}$ $\left(x_{j}, x_{k}^{\prime} \in T ; 1 \leq \forall j \leq m, 1 \leq \forall k \leq n\right)$, then $m=n$ and $x_{j}=x_{j}^{\prime}(1 \leq \forall j \leq m)$. It is easy to see that if $w$ can be written as the concatenation of elements of $T$ and $T$ satisfies the strict prefix property, then $w$ can be uniquely written. (Thus, provided that $T$ has the strict prefix property, the fact that $w$ can be written as the concatenation of elements of $T$ immediately implies that $T$ can parse $w$.)

Intuitively, the algorithm $L A$ behaves as follows. The algorithm maintains a set $T$ of edge labels that, at any time, is sufficient to parse all example strings seen so far. The goal of UPDATE is to update the set $T$ so that the new example can be parsed, while maintaining the parsability of all previously seen examples. Suppose that the first positive example is $a b b a$, the set $T$ consists only of the string $a b b a$ and clearly this is sufficient to parse itself. Now, if a new positive example is obtained, say $a b a a$, then $T=\{a b b a\}$ is not sufficient to parse abaa. Since the edge labels of the target SDA $M$ satisfy the strict prefix property, the edge label of $M$ that begins with $a$ must be a prefix of the longest common prefix of $a b b a$ and $a b a a$, i.e., a prefix of $a b$, in order for $M$ to parse both $a b b a$ and $a b a a$. Thus, in $T$ we replace $a b b a$ with $a b$. Now, the edge labels of $M$ must parse the corresponding suffixes $b a$ and $a a$ of $a b b a$ and $a b a a$, respectively, so UPDATE must ensure that both of these suffixes can be parsed. UPDATE maintains a queue of strings that need to be parsed in order for the final set $T$ to be able to parse the new string and all previously parsable strings.

In general, the set $T$ maintains a tentative set of edge labels of the SDA to be learned, and the elements of $T$ always satisfy the strict prefix property. At any time, elements of $T$ may be replaced, or they may be split into smaller pieces and new elements of $T$ may be added.

After updating $T$ with a new example, a procedure REFINE takes as input each transition from the currently conjectured SDA, and breaks it up into the new smaller parse strings of the updated set $T$, introducing a chain of new states if necessary. Then, a procedure PARSE adds into the current automaton a new chain of states (using the updated set $T$ of edge labels) that can exactly parse the new example.

Finally, CONSTRUCT takes the refined automaton, along with the new chain of states for the parse of the new example, and then condenses it into a deterministic automaton 
using Lemma 2.4 to merge equivalent states and edges.

Let $L$ be an SRL over $\Sigma=\left\{a_{1}, \ldots, a_{m}\right\}$ and $S_{i}=\left\{w_{1}, \cdots, w_{i}\right\}$ be the finite sample set of positive examples of $L$ provided up to the $i$-th stage of the learning process.

\section{(1) UPDATE}

We describe how the set $T$ is updated, when a new positive example $w$ is provided. Let $w_{i}$ be the $i$-th positive example provided at the $i$-th stage of the learning process and let $M_{i-1}=\left(Q_{i-1}, T_{i-1}, \delta_{i-1}, p_{0}, F_{i-1}\right)$ be the conjectured SDA at the $(i-1)$-st stage, where the set of strings of edge labels $T_{i}$ has the strict prefix property of Definition 5.

We now suppose that $w_{i}$ is not consistent with $M_{i-1}$, that is, $w_{i}$ is not in $L\left(M_{i-1}\right)$.

The procedure $\operatorname{UPDATE}\left(T_{i-1}, w_{i}\right)$ takes as input $T_{i-1}$ and $w_{i}$, and returns as output an updated set $T_{i}$ such that (1) $T_{i}$ can parse any string $w$ in $S_{i}$ (which includes the new string $w_{i}$ ), and (2) $T_{i}$ has the strict prefix property.

In describing the procedure, the following notations will be used :

- firstchar $(w)$ denotes the first symbol of $w$ if $w \neq \lambda$

- $\quad \operatorname{lcp}(u, v)$ denotes the longest common prefix of $u$ and $v$.

The procedure UPDATE is given below.

\section{[Correctness of UPDATE]}

From the way $T_{i-1}$ is updated, it is clear that $\operatorname{UPDATE}\left(T_{i-1}, w_{i}\right)$ always halts and returns an output set $T_{i}$. In order to prove the correctness of UPDATE, we must show that after the execution of UPDATE $\left(T_{i-1}, w_{i}\right)$ the following properties hold :

(1) $T_{i}$ can parse every string $w$ in $S_{i}$, and

(2) $T_{i}$ has the strict prefix property.

Let $w$ be a string from $Q$. As a notation, $w \Rightarrow^{x} w^{\prime}$ iff $w^{\prime}$ is a suffix of $w$ obtained by making one application of an operation $x(\in\{(\mathrm{a}),(\mathrm{b}),(\mathrm{c})\})$ of the case statements in the repeat loop. That is,

$$
w \Rightarrow^{x} w^{\prime} \text { iff }\left\{\begin{array}{l}
x=(\mathrm{a}) \text { and } w^{\prime}=\lambda \text { with } w \in T \\
x=(\mathbf{b}) \text { and } w=t_{a} w^{\prime} \text { with } t_{a} \in T, \\
x=(\mathrm{c}) \text { and } w=x_{a} w^{\prime} \text { and } x_{a}=\operatorname{lcp}\left(w, t_{a}\right) \text { with } x_{a} \in T .
\end{array}\right.
$$

The property (2) is shown by induction on $i$. For $i=1, T_{1}\left(=\left\{w_{1}\right\}\right)$ trivially has the strict prefix property. To see that $T_{i}\left(=\operatorname{UPDATE}\left(T_{i-1}, w_{i}\right)\right)$ has the strict prefix property, from the inductive hypothesis, we have only to observe that the strict prefix 
property of $T_{i-1}$ is preserved at any time through the operation of each case statement (a), (b) and (c) in the repeat loop, by construction.

Consider a sequence of operations : $w \Rightarrow^{x_{1}} w_{1} \Rightarrow^{x_{2}} \cdots \Rightarrow^{x_{n-1}} w_{n-1} \Rightarrow^{x_{n}} w_{n}(=\lambda)$. In this case, a word $x_{1} \cdots x_{n}\left(\in\{(\mathrm{a}),(\mathrm{b}),(\mathrm{c})\}^{+}\right)$is called the associate word of $u$.

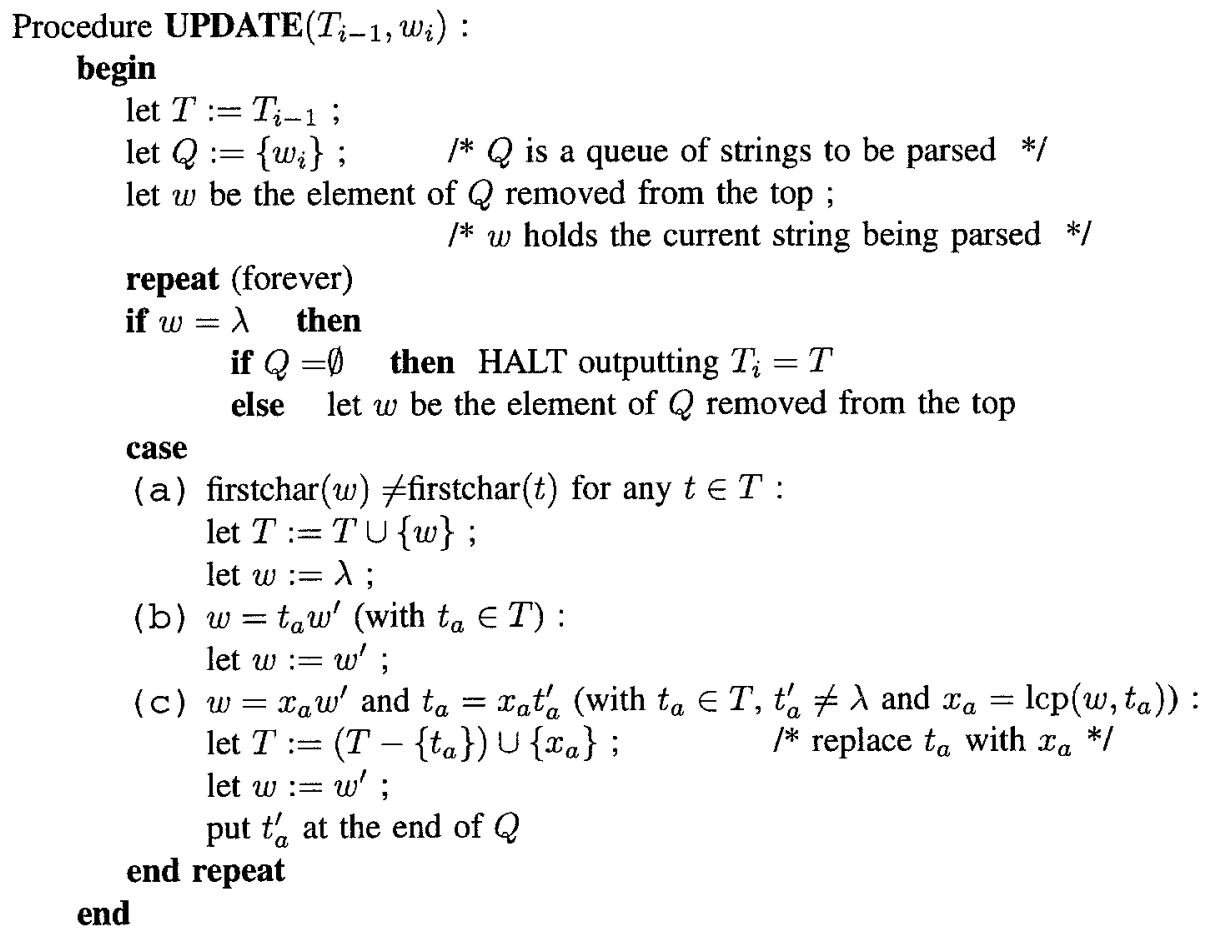

In order to prove the property (1) above, we first show the following claim :

[Claim] Every string $w$ ever put into $Q$ can be parsed by $T_{i}$.

Proof. Noting that every string $w$ in $Q$ is removed from the top of $Q$ to be scanned, let $\alpha$ be the associate word of $w$. We show by induction on $n=\operatorname{len}(\alpha)$ that $w$ can be parsed by the current set $T$ during the execution of $\operatorname{UPDATE}\left(T_{i-1}, w_{i}\right)$. For $n=1$, by construction, exactly one of the following three cases occurs : (i) $T:=T \cup\{w\}$ (in case (a)), (ii) $w=t_{a}$ with $t_{a} \in T$ (in case (b)), (iii) $w=x_{a}\left(=\operatorname{lcp}\left(w, t_{a}\right)\right) \in T$ (case (c)). Thus, in all cases, $w$ can be parsed by $T$. Suppose inductively that any string in $Q$ whose associate word has length less than $n$ can be parsed by $T$, and consider $w \Rightarrow^{x} w_{1} \Rightarrow^{\alpha^{\prime}} \lambda$ (for some $w_{1}$ ), where $\alpha=x \alpha^{\prime}$ is the associate word of $w$, $\operatorname{len}\left(\alpha^{\prime}\right)=n-1$ and $x \in\{(\mathrm{b}),(\mathrm{c})\}$. Then, by definition, either $w=t_{a} w_{1}$ with $t_{a} \in T$ or $w=x_{a} w_{1}$ with $x_{a} \in T$. By the inductive hypothesis, since $w_{1}$ can be parsed by $T$, we conclude that $w$ can be parsed by $T$. $T$ may be further updated by case (c) to finish scanning all strings in $Q$. In case (c), suppose that $t_{a}^{\prime}$ is put into $Q$, where 
$w=x_{a} w^{\prime}, t_{a}=x_{a} t_{a}^{\prime}$ and $t_{a}$ is replaced with $x_{a}$ in $T$. But, as argued above, since $t_{a}^{\prime}$ is parsed by the current $T, T$ can also parse $t_{a}$. Thus, we conclude that when UPDATE eventually terminates, $T_{i}$ can parse every string $w$ in $Q$. (End of the proof of Claim.)

We now prove the property (1) by induction on $i$. First, $T_{1}=\left\{w_{1}\right\}$ can trivially parse every string in $S_{1}\left(=\left\{w_{1}\right\}\right)$. Thus, we may assume as an induction hypothesis that $T_{i-1}$ can parse every string in $S_{i-1}$.

Suppose that $w$ is $w_{i}$ in $S_{i}\left(=S_{i-1} \cup\left\{w_{i}\right\}\right)$. Then, from the manner of constructing UPDATE, since $w$ is the first and only element of $Q$, the Claim implies that $T_{i}$ can parse $w$. Hence, we have only to show that $T_{i}$ can parse every string in $S_{i-1}$. But, to argue this, from the inductive hypothesis above, it suffices to show that $T_{i}$ can parse every string in $T_{i-1}$. This is clearly true for each element of $T_{i-1} \cap T_{i}$. Now, consider the case (c) that can only replace $t_{a}^{(i-1)}$ in $T_{i-1}$ with some prefix $t_{a}^{(i)}$ in $T_{i}$, where $t_{a}^{(i-1)}=t_{a}^{(i)} u$ and $u$ is put at the end of $Q$ to be later parsed. Then, by the Claim, $u$ can be parsed by $T_{i}$, and since $t_{a}^{(i-1)}$ can be written as the concatenation of $t_{a}^{(i)}$ and $u$, we eventually conclude that every $t_{a}^{(i-1)}$ in $T_{i-1}$ can be parsed by $T_{i}$. This completes the proof of the property (1).

(2) REFINE and PARSE

Let $T_{i}=\operatorname{UPDATE}\left(T_{i-1}, w_{i}\right)$ and $M_{i-1}=\left(Q_{i-1}, T_{i-1}, \delta_{i-1}, p_{0}, F_{i-1}\right)$ be the $(i-1)$-st conjecture. We describe how an SDA $M_{i}$ is constructed from $M_{i-1}$ and $T_{i}$. For that purpose, we need two subprocedures.

For a transition $t=(p, x, q) \in \delta_{i-1}$, we define $\operatorname{REFINE}\left(T_{i}, M_{i-1}, t\right)$ by the set of transitions

$$
\left\{\left(q_{0}, u_{1}, q_{1}\right),\left(q_{1}, u_{2}, q_{2}\right), \ldots,\left(q_{n-2}, u_{n-1}, q_{n-1}\right),\left(q_{n-1}, u_{n}, q_{n}\right)\right\},
$$

where $q_{0}=p, q_{n}=q, x=u_{1} \cdots u_{n}$, and for each $j(1 \leq j \leq n) u_{j}$ equals $t_{a}$ for some $t_{a} \in T_{i}$, and for each $j(1 \leq j \leq n-1) \quad q_{j}$ is newly introduced if a transition $\left(q_{j-1}, u_{j}, q_{j}\right)$ is not in $\delta_{i-1}$.

Further, define PARSE $\left(T_{i}, M_{i-1}, w_{i}\right)$ by the set of transitions

$$
\left\{\left(q_{0}, u_{1}, q_{1}\right),\left(q_{1}, u_{2}, q_{2}\right), \ldots,\left(q_{n-2}, u_{n-1}, q_{n-1}\right),\left(q_{n-1}, u_{n}, q_{n}\right)\right\},
$$

where $w_{i}=u_{1} \cdots u_{n}$, and for each $j(1 \leq j \leq n) u_{j}$ equals $t_{a}$ for some $t_{a} \in T_{i}$, and for each $j(1 \leq j \leq n) \quad q_{j}$ is newly introduced if a transition $\left(q_{j-1}, u_{j}, q_{j}\right)$ is not in $\delta_{i-1}$, and $q_{0}=p_{0}$ and $q_{n}$ is marked as a new accepting state if it is not in $F_{i-1}$. (That is, this entails the construction of $F_{i}$.)

(3) CONSTRUCT

Let $\Delta_{i}$ be the set of all transitions defined by

$$
\Delta_{i}=\bigcup_{t \in \delta_{i-1}} \operatorname{REFINE}\left(T_{i}, M_{i-1}, t\right) \cup \operatorname{PARSE}\left(T_{i}, M_{i-1}, w_{i}\right) .
$$


The procedure CONSTRUCT $\left(\Delta_{i}\right)$ takes as input $\Delta_{i}$ and returns a conjectured SDA $M_{i}$ by merging equivalent states and edges in $\Delta_{i}$ using Lemma 2.

Procedure CONSTRUCT $\left(\Delta_{i}\right)$ :

let $\Delta_{i}=\bigcup_{t \in \delta_{i-1}} \operatorname{REFINE}\left(T_{i}, M_{i-1}, t\right) \cup \operatorname{PARSE}\left(T_{i}, M_{i-1}, w_{i}\right)$;

merge identical states and edges in $\Delta_{i}$ using Lemma 2 ;

let $\delta_{i}$ be the result of merging states and edges from $\Delta_{i}$;

return $M_{i}=\left(Q_{i}, T_{i}, \delta_{i}, p_{0}, F_{i}\right)$, where $Q_{i}, F_{i}$ are obtained

from $\delta_{i}$ in the obvious manner.

The complete algorithm $L A$ is given below.

\subsection{Correctness of $L A$}

In this section we will prove the correctness of the presented algorithm $L A$. To this end, we start by defining an operation which is useful for our purpose.

Define a binary operation $\mathcal{O}$ on strings as follows : for $x, y \in \Sigma^{*}$,

$$
\mathcal{O}(x, y)=\{u, v, w\}, \text { where } u=\operatorname{lcp}(x, y), x=u v, y=u w
$$

(Recall that $\operatorname{lcp}(x, y)$ denotes the longest common prefix of $x$ and $y$.) Further, for a finite set of strings $S$, extend $\mathcal{O}$ by

$$
\mathcal{O}(S)=\bigcup_{(x, y) \in S \times S} \mathcal{O}(x, y)
$$

Then, consider the closure of $S$ under $\mathcal{O}$ and denote it by $\mathcal{O}^{*}(S)$, i.e., let $\mathcal{O}^{*}(S)=$ $\bigcup_{n \geq 0} \mathcal{O}^{n}(S)$, where $\mathcal{O}^{0}(S)=S$ and $\mathcal{O}^{n+1}(S)=\mathcal{O}\left(\mathcal{O}^{n}(S)\right.$ ) (for each $n \geq 0$ ).

Let $F$ be a finite set of strings over $\Sigma^{*}$ where every symbol of $\Sigma$ appears in some string in $F$. For each $a \in \Sigma, F_{a}$ denotes the set of strings in $F$ that start with $a$. Further, by min $F$ we denote $\left\{\operatorname{sh}\left(F_{a}\right) \mid a \in \Sigma\right\}$, where each $\operatorname{sh}\left(F_{a}\right)$ is the set of shortest strings in $F_{a}$. (If $\operatorname{sh}\left(F_{a}\right)$ is a singleton $\left\{f_{a}\right\}$, then we simply write $f_{a}$ rather than $\left\{f_{a}\right\}$.)

We now claim that for each $a \in \Sigma$, if $\mathcal{O}^{*}(S)_{a}$ is not empty, then there is a unique shortest string $x_{a}$ in it, i.e., $\operatorname{sh}\left(\mathcal{O}^{*}(S)_{a}\right)=x_{a}$. Suppose there are shortest strings $x_{1}$ and $x_{2}$ in $\mathcal{O}^{*}(S)_{a}$ such that $x_{1}=u_{a} v, x_{2}=u_{a} w, \operatorname{len}(v)=\operatorname{len}(w)$ and $u_{a}=\operatorname{lcp}\left(x_{1}, x_{2}\right)$. If $v \neq \lambda$, then len $\left(u_{a}\right)<\operatorname{len}\left(x_{1}\right)$ and $u_{a} \in \mathcal{O}^{*}(S)_{a}$, contradicting the shortestness of $x_{1}$ in $\mathcal{O}^{*}(S)_{a}$. Hence, it must hold that $v=\lambda(=w)$, and we have that $x_{1}=x_{2}$, thus proving the claim. 


\section{[Learning algorithm $L A]$}

Input: a positive presentation of a strictly regular language $L$.

Output: a sequence of SDAs for strictly regular languages.

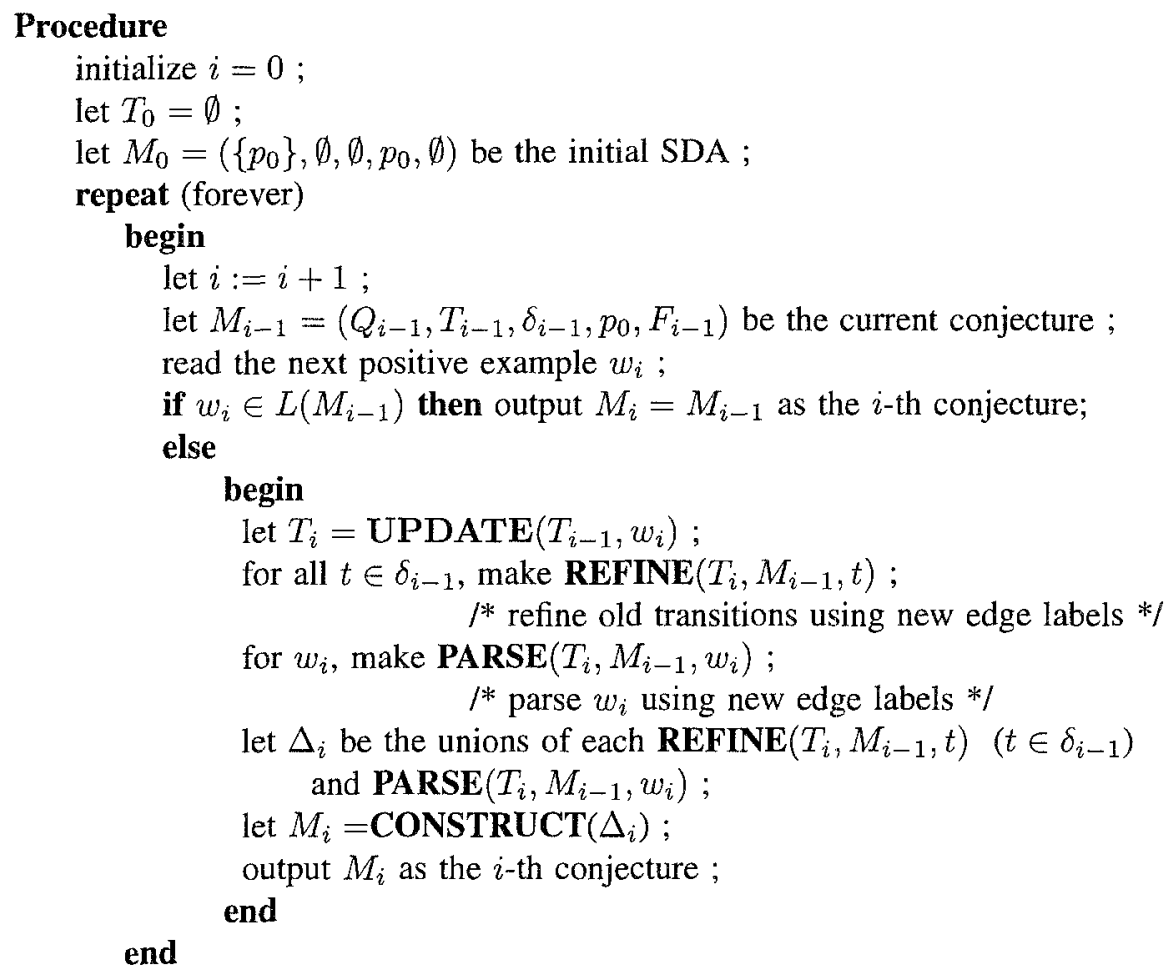

For each $i \geq 1$, let $S_{i}=\left\{w_{1}, \ldots, w_{i}\right\}$ be a sample set of a target SRL $L$. Let $T_{S_{i}}=\min \mathcal{O}^{*}\left(S_{i}\right)=\left\{x_{a} \mid a \in \Sigma\right\}$, where for each $a \in \Sigma, x_{a}=\operatorname{sh}\left(\mathcal{O}^{*}\left(S_{i}\right)_{a}\right) . T_{S_{i}}$ clearly has the strict prefix property. Also, from the strict prefix property of $T_{S_{i}}$, it is easy to show that any string $w$ in $S_{i}$ can be parsed by $T_{S_{i}}$, i.e., $w$ can be written as the concatenation of elements of $T_{S_{i}}$ with the property that $w=x_{1} \cdots x_{m}=x_{1}^{\prime} \cdots x_{n}^{\prime}$ $\left(x_{j}, x_{k}^{\prime} \in T_{S_{i}} ; 1 \leq \forall j \leq m, 1 \leq \forall k \leq n\right)$ implies that $m=n$ and $x_{j}=x_{j}^{\prime}$ $(1 \leq \forall j \leq m)$.

Using this formalization, $T_{i}\left(=\operatorname{UPDATE}\left(T_{i-1}, w_{i}\right)\right)$ is characterized by $T_{S_{i}}$ as follows

Lemma 6 Let $S_{i}=\left\{w_{1}, \ldots, w_{i}\right\}$. Then, for each $i \geq 1, T_{S_{i}}$ coincides with $T_{i}$. 
Proof: By induction on $i$. Suppose $i=1$, then $S_{1}=\left\{w_{1}\right\}$ and $T_{0}=\emptyset$. Since $\mathcal{O}\left(S_{1}\right)=\left\{\lambda, w_{1}\right\}=\{\lambda\} \cup S_{1}$, we have that $\mathcal{O}^{*}\left(S_{1}\right)=\{\lambda\} \cup S_{1}$. Hence, $T_{S_{1}}=\left\{x_{a}\right\}=$ $T_{1}\left(=\operatorname{UPDATE}\left(T_{0}, w_{1}\right)\right)$, where $x_{a}=w_{1}$ and $a$ is the first symbol of $w_{1}$.

Since $S_{i-1} \subseteq \mathcal{O}^{*}\left(S_{i-1}\right)$, it holds that $\mathcal{O}^{*}\left(S_{i-1} \cup\left\{w_{i}\right\}\right) \subseteq \mathcal{O}^{*}\left(\mathcal{O}^{*}\left(S_{i-1}\right) \cup\left\{w_{i}\right\}\right)$. Conversely, since $\mathcal{O}^{*}\left(S_{i-1}\right) \cup\left\{w_{i}\right\} \subseteq \mathcal{O}^{*}\left(S_{i}\right)$, we have that $\mathcal{O}^{*}\left(\mathcal{O}^{*}\left(S_{i-1}\right) \cup\left\{w_{i}\right\}\right) \subseteq$ $\mathcal{O}^{*}\left(\mathcal{O}^{*}\left(S_{i}\right)\right)=\mathcal{O}^{*}\left(S_{i}\right)=\mathcal{O}^{*}\left(S_{i-1} \cup\left\{w_{i}\right\}\right)$. Thus, it is proved that

$$
\min \mathcal{O}^{*}\left(S_{i-1} \cup\left\{w_{i}\right\}\right)=\min \mathcal{O}^{*}\left(\mathcal{O}^{*}\left(S_{i-1}\right) \cup\left\{w_{i}\right\}\right) .
$$

Suppose now that $T_{S_{i-1}}=T_{i-1}$, in other words, suppose that

$$
T_{i-1}=\min \mathcal{O}^{*}\left(S_{i-1}\right) \quad \cdots \quad \text { (inductive hypothesis) } .
$$

By construction, since every string in $\mathcal{O}^{*}\left(S_{i-1}\right)$ can be parsed by $T_{i-1}\left(=\min \mathcal{O}^{*}\left(S_{i-1}\right)\right)$, it holds that

$$
\min \mathcal{O}^{*}\left(\mathcal{O}^{*}\left(S_{i-1}\right) \cup\left\{w_{i}\right\}\right)=\min \mathcal{O}^{*}\left(\min \mathcal{O}^{*}\left(S_{i-1}\right) \cup\left\{w_{i}\right\}\right) .
$$

Then, noting that $T_{i}=\min \mathcal{O}^{*}\left(T_{i-1} \cup\left\{w_{i}\right\}\right)$, we have

$$
\begin{array}{rlr}
T_{S_{i}} & =\min \mathcal{O}^{*}\left(S_{i}\right) & \\
& =\min \mathcal{O}^{*}\left(S_{i-1} \cup\left\{w_{i}\right\}\right) & \\
& =\min \mathcal{O}^{*}\left(\mathcal{O}^{*}\left(S_{i-1}\right) \cup\left\{w_{i}\right\}\right) & \text { (by (1)) } \\
& =\min \mathcal{O}^{*}\left(\min \mathcal{O}^{*}\left(S_{i-1}\right) \cup\left\{w_{i}\right\}\right) & \text { (by (2)) } \\
& =\min \mathcal{O}^{*}\left(T_{i-1} \cup\left\{w_{i}\right\}\right) & \text { (by inductive hypothesis) } \\
& =T_{i} . &
\end{array}
$$

Thus, the lemma is proved.

We also show that $T_{i}$ is the "coarsest" finite set in the following sense.

Lemma 7 Let $T_{i}=T_{S_{i}}$ and let $T^{\prime}$ be any finite set of strings over $\Sigma$ with the strict prefix property and such that every string in $S_{i}$ can be parsed by $T^{\prime}$. Then, every element in $T_{i}$ can be parsed by $T^{\prime}$.

Proof: Since the $\mathcal{O}(X)$ operator adds only shorter strings to a set $X$, there is some finite $k$ such that $\mathcal{O}^{*}\left(S_{i}\right)=\mathcal{O}^{k}\left(S_{i}\right)$. Now we show by induction on $n$ that $T^{\prime}$ can parse every string in $\mathcal{O}^{n}\left(S_{i}\right)$. Then, since $T_{i}=T_{S_{i}}=\min \mathcal{O}^{*}\left(S_{i}\right)=\min \mathcal{O}^{k}\left(S_{i}\right)$, this immediately implies that every element in $T_{i}$ can be parsed by $T^{\prime}$.

By the hypothesis of the lemma, $T^{\prime}$ parses every string in $S_{i}=\mathcal{O}^{0}\left(S_{i}\right)$. Assume inductively that it parses every string in $\mathcal{O}^{n}\left(S_{i}\right)$. Then, the only strings in $\mathcal{O}^{n+1}\left(S_{i}\right)-$ $\mathcal{O}^{n}\left(S_{i}\right)$ are triples of strings $u, v$ and $w$ such that $u v$ and $u w$ are in $\mathcal{O}^{n}\left(S_{i}\right)$, and the first characters of $v$ and $w$ are different. Thus, for such strings, $T^{\prime}$ parses $u v$ and $u w$ by the inductive hypothesis. Since $T^{\prime}$ has the strict prefix property, it is now not difficult to 
show that $T^{\prime}$ parses each of $u, v$ and $w$, thus completing the proof.

We are now in a position to prove the following lemma.

LEMMA 8 For each $i \geq 1, M_{i}$ accepts the smallest SRL containing $S_{i}$. That is, $S_{i} \subseteq L\left(M_{i}\right)$ and for each $i \geq 1$, if $L^{\prime}$ is an SRL containing $S_{i}$, then $L\left(M_{i}\right) \subseteq L^{\prime}$.

Proof: Let $M_{i}=\left(Q_{i}, T_{i}, \delta_{i}, p_{0}, F_{i}\right)$ be the $i$-th conjecture. Then, by construction, it holds that $S_{i} \subseteq L\left(M_{i}\right)$. Let $M^{\prime}=\left(Q^{\prime}, T^{\prime}, \delta^{\prime}, p_{0}^{\prime}, F^{\prime}\right)$ be an acceptor such that $L^{\prime}=L\left(M^{\prime}\right)$ containing $S_{i}$. By Lemma 7 , each element of $T_{i}$ can be written as a concatenation of elements of $T^{\prime}$. Consider any $u$ that is a prefix of a string $w$ in $S_{i}$ and is such that $u$ can be written as a concatenation of elements of $T_{i}$. Then, since $M^{\prime}$ accepts $w$ and $u$ is a prefix of $w$ that can be written as a concatenation of elements of $T_{i}, \delta^{\prime}\left(p_{0}^{\prime}, u\right)$ is a state in $Q^{\prime}$.

Now, define a mapping $f$ from $Q_{i}$ to $Q^{\prime}$ as follows: For each $p \in Q_{i}$, let $u_{p}$ be a prefix of a string in $S_{i}$ such that the number of transitions in $\delta_{i}\left(p_{0}, u_{p}\right)=p$ is minimum. Then, define $f(p)=\delta^{\prime}\left(p_{0}^{\prime}, u_{p}\right)$. (As seen above, this is well-defined.) We show the following : (1) $f\left(p_{0}\right)=p_{0}^{\prime},(2)$ if $p$ is in $F_{i}$, then $f(p)$ is in $F^{\prime}$, (3) for every $p \in Q_{i}$ and every label string $t$ such that $\delta(p, t)=q$ for some $q, f\left(\delta_{i}(p, t)\right)=\delta^{\prime}(f(p), t)$.

By letting $u_{p_{0}}=\lambda$ and from the definitions of $f$ and $\delta^{\prime}$, we have

$$
f\left(p_{0}\right)=\delta^{\prime}\left(p_{0}^{\prime}, u_{p_{0}}\right)=\delta^{\prime}\left(p_{0}^{\prime}, \lambda\right)=p_{0}^{\prime} .
$$

Suppose that $p$ is in $F_{i}$. Then, since $u_{p}$ is a prefix of a string in $S_{i}$, there exists a string $v$ such that $u_{p}$ and $u_{p} v$ are in $L\left(M_{i}\right)$ and that $u_{p} v$ is in $S_{i}$, where $\delta_{i}(p, v) \in F_{i}$. Let $w_{p}$ be a string in $S_{i}$ such that $\delta_{i}\left(p_{0}, w_{p}\right)=p \in F_{i}$. From the manner $F_{i}$ is constructed using $S_{i}$ and the minimality of $u_{p}, u_{p}$ is in $L\left(M_{i}\right)$ implies that $u_{p}=w_{p}$ and, therefore, $u_{p}$ is in $S_{i}$. Since $S_{i}$ is contained in $L^{\prime}$, we have that $u_{p}$ is in $L^{\prime}$. That is, $f(p)=\delta^{\prime}\left(p_{0}^{\prime}, u_{p}\right)$ is in $F^{\prime}$.

To prove (3), since $t$ is a label string in $T_{i}$ and $t$ can be written as a concatenation of elements of $T^{\prime}$, there exists a state $\delta^{\prime}(f(p), t)$ in $Q^{\prime}$. For $q \in Q_{i}$ such that $\delta_{i}(p, t)=q$, let $u_{q}=u_{p} t$. Then, from the manner of choosing $u_{p}, u_{q}$ is a prefix of a string in $S_{i}$ such that the number of transitions in $\delta_{i}\left(p_{0}, u_{q}\right)\left(=\delta_{i}\left(p_{0}, u_{p} t\right)\right)=q$ is minimum. Further, from the manner $\delta_{i}$ is constructed, there is a string in $S_{i}$ whose prefix is $u_{q}$. Therefore, we have that $f(q)=\delta^{\prime}\left(p_{0}^{\prime}, u_{q}\right)$. Then,

$$
\begin{aligned}
\delta^{\prime}(f(p), t) & =\delta^{\prime}\left(\delta^{\prime}\left(p_{0}^{\prime}, u_{p}\right), t\right) \\
& =\delta^{\prime}\left(p_{0}^{\prime}, u_{p} t\right) \\
& =\delta^{\prime}\left(p_{0}^{\prime}, u_{q}\right) \\
& =f(q) \\
& =f\left(\delta_{i}(p, t)\right) .
\end{aligned}
$$


Thus, (1), (2) and (3) above immediately imply that $L\left(M_{i}\right) \subseteq L^{\prime}$.

As a corollary, we have

COROLLARY 9 Let $L$ be a target $S R L$. Then, for each $i \geq 1$, it holds that $L\left(M_{i-1}\right) \subseteq$ $L\left(M_{i}\right) \subseteq L$.

In order to prove the correctness of the algorithm $L A$, we need to introduce the notion of a characteristic sample for a target SRL $L$. A finite subset $S$ of $L$ is called a characteristic sample of $L$ if and only if $L$ is the smallest SRL containing $S$, i.e., for any SRL $L^{\prime}, S \subseteq L^{\prime}$ implies that $L \subseteq L^{\prime}$ (Angluin, 1982).

Corollary 9 together with the definition of a characteristic sample almost immediately implies the following.

LEMma 10 For any $i \geq 1$, if $S_{i}$ is a characteristic sample of $L$, then $L=L\left(M_{i}\right)$. Further, for any $S$ if $S$ is a characteristic sample of $L$, then so is any $S_{i}$ containing $S$.

We will show that there effectively exists a characteristic sample $S_{L}$ of each SRL $L$.

We say that $M$ is in normal form iff (1) it has no useless states, (2) every accepting sink state has exactly one transition defined into it, and (3) for every non-accepting state which is not the initial state, there are at least two transitions defined from it, where sink state is a state from which no transitions are defined.

Using an example, we demonstrate that SDAs in this form actually provide "normal forms" for SDAs.

Example 3 Consider an SDA $M$ given in Figure 3. $M$ has no useless states. For an accepting sink state $p_{4}$ that violates (2), duplicate it and introduce new state $p_{4}^{\prime}$. For non-accepting states $p_{1}$ and $p_{3}$ that violate (3) and are not the initial state, we "haul up" each chain of transitions until the condition (3) holds, and delete $p_{1}$ and $p_{3}$ and, finally, replace old transitions involved in them with new transitions $\left(p_{0}, a b, p_{2}\right),\left(p_{2}, d b, p_{2}\right)$ and $\left(p_{2}, c f, p_{4}^{\prime}\right)$. Thus, a normal form $M^{\prime}$ is obtained such that $M^{\prime}$ is equivalent to $M$.

It should be clear that any SDA can be transformed into such a normal form without changing the language.

Let $M=\left(Q, \Sigma, \delta, p_{0}, F\right)$ be an SDA in normal form that accepts $L$. We now construct a finite set $S_{M}$ as follows. If $M$ accepts only one string $w$, then let $S_{M}=\{w\}=L(M)$. Otherwise, we proceed below.

For each $p \in Q$, define the string $u_{p}$ such that $\delta\left(p_{0}, u_{p}\right)=p$ in the following manner:

1. $u_{p_{0}}=\lambda$,

2. for any state $p$ that is non-accepting or is not a sink state, let $u_{p}$ be any string such that $\delta\left(p_{0}, u_{p}\right)=p$, 

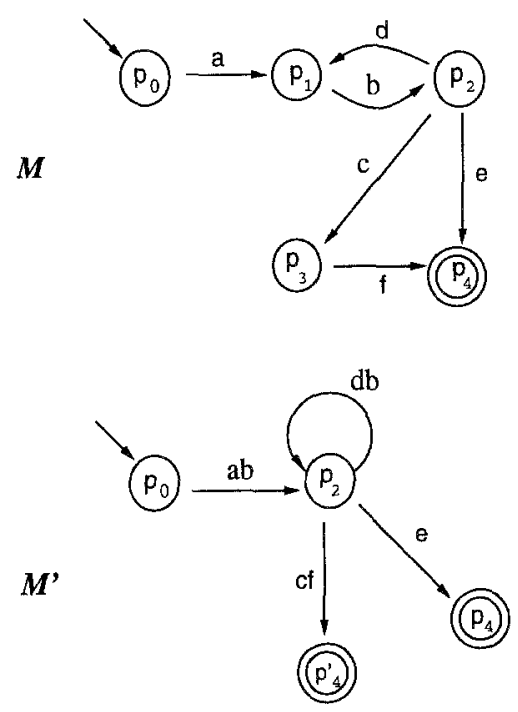

Figure 3. $M$ and Its Normal Form $M$ I

3. for each accepting sink state $q$ such that $\delta(p, t)=q$, let $u_{q}=u_{p} t$, where $t$ is the unique label of a transition from $p$ into $q$.

Also, for each $p \in Q$, we choose any string $v_{p}$ such that $\delta\left(p, v_{p}\right) \in F$. Finally, for each $q \in Q$, we define a set $X_{q}$ consisting of one or two strings as follows:

(1) if $q$ is an accepting sink state, then $X_{q}=\{\lambda\}$,

(2) if $q$ is an accepting state with at least one transition defined from it, then choose one such transition $(q, t, p)$ in $\delta$, and $X_{q}=\left\{\lambda, t v_{p}\right\}$,

(3) if $q$ is a non-accepting state (hence by the normal form, there are at least two transitions defined from it), then choose distinct transitions $\left(q, t_{1}, q_{1}\right)$ and $\left(q, t_{2}, q_{2}\right)$ in $\delta$ and let $X_{q}=\left\{t_{1} v_{q_{1}}, t_{2} v_{q_{2}}\right\}$,

(4) finally, if the initial state $p_{0}$ is non-accepting and has nothing but one transition defined from it, then $X_{p_{0}}=\left\{v_{p_{0}}\right\}$.

Now, we define a finite set $S_{M}$ as follows :

$$
S_{M}=\bigcup_{p \in Q} u_{p} X_{p} \cup \bigcup_{(p, t, q) \in \delta} u_{p} t X_{q}
$$


Example 4 Consider the SDA $M^{\prime}$ in Example 3. For each $p \in Q$, we choose $u_{p}$ and $v_{p}$ as follows:

$$
\begin{aligned}
& u_{p_{0}}=\lambda, \quad u_{p_{2}}=a b, u_{p_{4}}=a b e, u_{p_{4}^{\prime}}=a b c f \\
& v_{p_{0}}=a b e, \quad v_{p_{2}}=e, \quad v_{p_{4}}=\lambda, \quad v_{p_{4}^{\prime}}=\lambda \text {. }
\end{aligned}
$$

(Note that we could instead choose $u_{p_{2}}=a b d b, u_{p_{4}}=a b d b e, u_{p_{4}^{\prime}}=a b d b c f$.)

Then, construct

$$
X_{p_{0}}=\{a b e\}, X_{p_{2}}=\{e, c f\}, X_{p_{4}}=\{\lambda\}, X_{p_{4}^{\prime}}=\{\lambda\} .
$$

Finally, define $S_{M^{\prime}}$ by

$$
\begin{aligned}
S_{M^{\prime}}= & \{a b e\} \cup\{a b e, a b c f\} \cup\{a b e\} \cup\{a b c f\} \\
& \cup\{a b e, a b c f\} \cup\{a b d b e, a b d b c f\} \cup\{a b e\} \cup\{a b c f\} \\
= & \{a b e, a b c f, a b d b e, a b d b c f\} .
\end{aligned}
$$

LEMMA 11 For any SDA $M, S_{M}$ is a characteristic sample of $L(M)$.

Proof: By construction, $S_{M} \subseteq L(M)$. We may assume that $M$ accepts at least two strings. Let $M^{\prime}$ be the SDA $M_{S_{M}}$ produced by $L A$ when $S_{M}$ is provided as a sample set. As we have seen in Lemma 8, since $L\left(M^{\prime}\right)$ is the smallest SDL containing $S_{M}$, we have that $L\left(M^{\prime}\right) \subseteq L(M)$. Suppose it is proved that $L(M) \subseteq L\left(M^{\prime}\right)$, then we have that $L(M)=L\left(M^{\prime}\right)$, which implies that $L(M)$ is the smallest SDL containing $S_{M}$. Therefore, it suffices to show that $L(M)$ is contained in $L\left(M^{\prime}\right)$.

Let $M=\left(Q, T, \delta, p_{0}, F\right)$ and $M^{\prime}=\left(Q^{\prime}, T^{\prime}, \delta^{\prime}, p_{0}^{\prime}, F^{\prime}\right)$ be SDAs described above. We show the following :

[Claim] For each $p \in Q$ and each $t$ such that $(p, t, q) \in \delta$ for some $q \in Q$, both strings $u_{p}$ and $u_{p} t$ lead to states of $M^{\prime}$ from $p_{0}^{\prime}$.

(Proof.) If $p$ is an accepting state, then $u_{p}$ is clearly in $S_{M}$ and, hence, in $L\left(M^{\prime}\right)$. Thus, $u_{p}$ leads to a state of $M^{\prime}$. If $p$ is neither the initial nor an accepting state, then there are two strings $u_{p} t_{1} v_{q_{1}}$ and $u_{p} t_{2} v_{q_{2}}$ in $S_{M}\left(\subseteq L\left(M^{\prime}\right)\right)$, where $t_{1}$ and $t_{2}$ begin with different symbols. Since both are accepted by $M^{\prime}$, the longest common prefix of the two, $u_{p}$, leads to a state of $M^{\prime}$. (Recall that if strings $x$ and $y$ are accepted by an SDA $M$, then the longest common prefix of $x$ and $y$ reaches a state of $M$ from the initial state.) If $p$ is the initial state $p_{0}$, then since $u_{p_{0}}=\lambda$, by definition, $u_{p_{0}}$ trivially leads to a state $\left(p_{0}^{\prime}\right)$ of $M^{\prime}$.

Further, suppose that $p$ has a transition $(p, t, q)$, then from the manner $S_{M}$ is constructed, either there is a string or there are two strings of the form $u_{p} t v_{q}$ in $u_{p} t X_{q}(\subseteq$ $S_{M} \subseteq L\left(M^{\prime}\right)$ ). If $q$ is an accepting state, then one can take $v_{q}=\lambda$ from $X_{q}$ and 
$u_{p} t\left(=u_{p} t v_{q}\right)$ is in $L\left(M^{\prime}\right)$. Thus, $u_{p} t$ leads to a state of $M^{\prime}$. If $q$ is neither the initial nor an accepting state, then there are two strings $v_{1}, v_{2}$ in $X_{q}$ such that they begin with different symbols. Since $u_{p} t v_{1}$ and $u_{p} t v_{2}$ are accepted by $M^{\prime}$, the longest common prefix $u_{p} t$ of these two leads to a state of $M^{\prime}$. Finally, if $q$ is the initial state $p_{0}$ of $M$, then there is a non-empty string $v_{p_{0}}$ in $X_{p_{0}}$ such that $u_{p} t v_{p_{0}}$ is in $S_{M}\left(\subseteq L\left(M^{\prime}\right)\right)$. By definition, since $v_{p_{0}}$ is in $S_{M}$, it is also in $L\left(M^{\prime}\right)$. From Corollary 3 , this implies that $u_{p} t$ leads to a state $p_{0}^{\prime}$ of $M^{\prime}$ (End of the proof of Claim).

We now define a mapping $f$ as follows : for each $p \in Q, f(p)=\delta^{\prime}\left(p_{0}^{\prime}, u_{p}\right)$. We would like to verify that (1) $f\left(p_{0}\right)=p_{0}^{\prime}$, (2) if $p \in Q$ is an accepting state, then so is $f(p)$, and (3) for each transition $(p, t, q), f(\delta(p, t))=\delta^{\prime}(f(p), t)$. Since, by definition, $u_{p_{0}}=\lambda$, (1) obviously holds. If $p$ is in $F$, then $u_{p}$ is in $S_{M}$, and hence, in $L\left(M^{\prime}\right)$. Thus, $f(p)\left(=\delta^{\prime}\left(p_{0}^{\prime}, u_{p}\right)\right)$ is in $F^{\prime}$.

To show (3), it is sufficient to show that $f(q)=\delta^{\prime}(f(p), t)$, since

$$
\begin{aligned}
f(\delta(p, t)) & =f\left(\delta\left(p_{0}, u_{p} t\right)\right) \\
& =f\left(\delta\left(p_{0}, u_{q}\right)\right) \\
& =f(q) .
\end{aligned}
$$

To show that $f(q)=\delta^{\prime}(f(p), t)$, we may equivalently show that $\delta^{\prime}\left(p_{0}^{\prime}, u_{p} t\right)=\delta^{\prime}\left(p_{0}^{\prime}, u_{q}\right)$.

Consider the cases for the state $q \in Q$ : if $q$ is an accepting sink state, then by definition, $u_{q}=u_{p} t$. Thus, the identity relation to be proved holds trivially. Otherwise, i.e., if $q$ is a state with at least one transition defined from it, then $X_{q}$ contains at least one non-empty string $v^{\prime}$ such that $\delta\left(q, v^{\prime}\right) \in F$. Then, there are two strings $u_{q} v^{\prime}$ and $u_{p} t v^{\prime}$ in $S_{M}\left(\subseteq L\left(M^{\prime}\right)\right)$. Since $M^{\prime}$ accepts these two strings, from Corollary 3 , we have that $\delta^{\prime}\left(p_{0}^{\prime}, u_{q}\right)=\delta^{\prime}\left(p_{0}^{\prime}, u_{p} t\right)$, completing the proof that (3) holds.

From these properties (1), (2) and (3), we conclude that $L(M) \subseteq L\left(M^{\prime}\right)$ holds, which completes the proof of Lemma 11.

We are now in a position to prove the following theorem.

THEOREM 12 The algorithm LA learns the class of SDAs in the limit from positive data.

Proof: We prove that the algorithm $L A$ converges to a correct SDA $M$ accepting the target language $L$. We claim the following:

[Claim] For a characteristic sample $S_{L}$ of $L$, if $S_{L} \subseteq S_{i_{0}}$ for some $i_{0}$, then $L=L\left(M_{i_{0}}\right)$. From Lemma 10, when the sample set $S_{i_{0}}$ contains a characteristic sample $S_{L}, S_{i_{0}}$ is also a characteristic sample of $L$. Then, from the property of a characteristic sample of $L$, it holds that $L \subseteq L\left(M_{i_{0}}\right)$. On the other hand, by Corollary 9, we have that for each $i \geq 1, L\left(M_{i}\right) \subseteq L$, and hence, we conclude that $L=L\left(M_{i_{0}}\right)$. Thus, the claim holds. Since $S_{L}$ is finite, there exists $i_{0} \geq 1$ such that $S_{L} \subseteq S_{i_{0}}$. From the claim, this completes the proof. 


\subsection{Time Analysis of $L A$}

We analyze the time complexity of the algorithm $L A$.

\section{[1] Time for Updating Conjectures}

For each $i \geq 1$, let $M_{i}=\left(Q_{i}, T_{i}, \delta_{i}, p_{0}, F_{i}\right)$ be the SDA produced by $L A$ and $S_{i}$ be the set of positive examples at the $i$-th stage. Each time a new positive example $w_{i}$ is given, updating the previous conjecture $M_{i-1}$ is performed in time at most $\mathrm{O}(\ell m)$, where $\ell=\operatorname{Max}_{w \in S_{i}}\{\operatorname{len}(w)\}, m=|\Sigma|$. This is proved as follows :

(1) First, we consider UPDATE $\left(T_{i-1}, w_{i}\right)$. An important observation about UPDATE is that only string matching operations are performed and nothing else is used throughout the procedure. Hence, all we have to do is to analyse how many lengths of strings, in total, must be scanned by UPDATE. Recall the construction of UPDATE. Each time case (c) occurs, some $t_{a}$ in $T$ is replaced with a proper suffix. (See Figure 4.) Thus, the number of times case (c) can occur is bounded by $x=\sum_{t_{a} \in T} l e n\left(t_{a}\right)$. Hence, the sum of the lengths of all of the strings ever put into $Q$ (except for $w_{i}$ ) is at most $x$. Note that the length of each element of $T$ is bounded by $\ell$.

The algorithm halts after all strings ever put into $Q$ (including the input string $w_{i}$ ) have been scanned. At each iteration of the repeat loop, at least one character of one of these strings is scanned as a prefix of the current string $w$ in case (a), (b), or (c). Hence, the total number of iterations of the repeat loop is at most $\operatorname{len}\left(w_{i}\right)+x$.

Thus, the total complexity of $\operatorname{UPDATE}\left(T_{i-1}, w_{i}\right)$ is bounded by:

$$
\begin{gathered}
\operatorname{len}\left(w_{i}\right)+\sum_{t_{a} \in T_{i-1}} \operatorname{len}\left(t_{a}\right) \\
\leq \mathrm{O}(\ell m), \text { where } \ell=\operatorname{Max}_{w \in S_{i}}\{\operatorname{len}(w)\} .
\end{gathered}
$$

(2) First, for each $t=(p, u, q) \in \delta_{i-1}\left(u \in T_{i-1}\right), \operatorname{REFINE}\left(T, M_{i-1}, t\right)$ is performed in time linear in $\operatorname{len}(u)$. For $\operatorname{PARSE}\left(T, M_{i-1}, w_{i}\right)$, it requires at most linear time in $l e n\left(w_{i}\right)$. Thus, REFINE and PARSE require at most $\ell m+l e n\left(w_{i}\right)$.

In order to check if two transitions $(p, u, q),\left(p^{\prime}, u^{\prime}, q^{\prime}\right) \in \Delta_{i}$ are mergable or not, we have only to check the first symbols of $u$ and $u^{\prime}$. Since the cardinality of $\Delta_{i}$ is bounded by $\ell m+\operatorname{len}\left(w_{i}\right)$, CONSTRUCT requires at most $\ell m+\operatorname{len}\left(w_{i}\right)$.

Hence, we have the following :

THEOREM 13 The algorithm $L A$ may be implemented to run in time $O(|\Sigma| \ell)$, where $\ell$ is the maximum length of all positive data provided.

\section{[2] A Bound on the Number of Implicit Prediction Errors}

We now consider the number of implicit errors of prediction needed for learning a correct SDA $M$ accepting the target SRL $L$. First, we define the size of an SDA $M=\left(Q, T, \delta, p_{0}, F\right)$ based on the sum of the lengths of all elements in $T$, that is, 


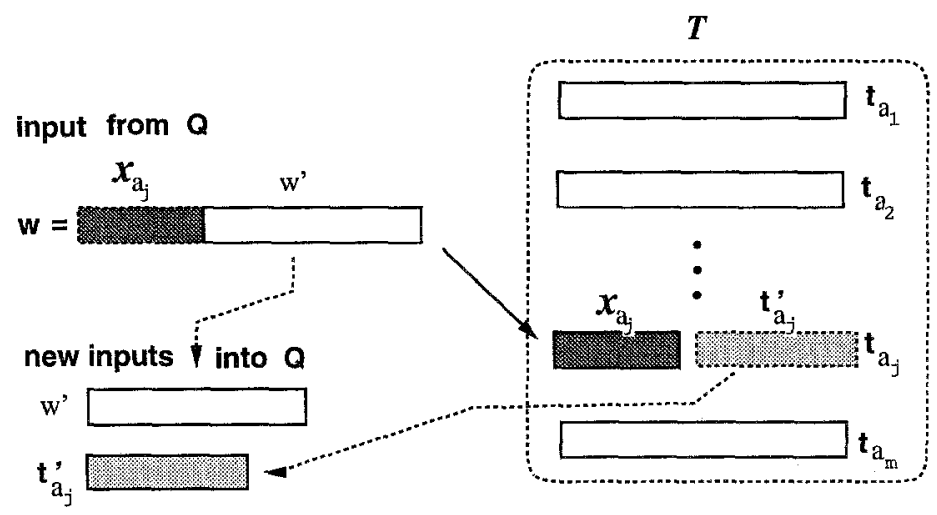

Figure 4. Updating $T$ in UPDATE(T,w)

$\operatorname{size}(M)=\sum_{u \in T}(l e n(u)+1)$. This may be justified when we think of an equivalent minimum DFA in the usual sense.

LEMMA 14 The number of implicit errors of prediction LA makes is at most $O(|\Sigma| \ell)$, where $\ell$ is the maximum length of all positive data provided.

Proof: Let $M$ be a correct SDA for the target $L$, and for each $i \geq 1$, let $M_{i}$ be the $i$-th conjecture. Each time $L A$ makes an implicit error of prediction, let us see what can happen to the conjecture in the algorithm $L A$. Suppose that $w\left(=w_{i}\right)$ is a positive example inconsistent with the previous conjecture $M_{i-1}$, i.e., $w_{i} \notin L\left(M_{i-1}\right)$. First, since the set of transitions of each conjecture contains at most $|\Sigma|$ elements of the form $(p, u, q)$, the maximum number of distinct states of any conjecture is bounded by $2|\Sigma|$. The following are possible cases where some changes to $M_{i-1}$ must be made to produce a new conjecture $M_{i}$ :

1. The set $T$ is unchanged and exactly one non-accepting state of $M_{i-1}$ becomes an accepting state of $M_{i}$. This can happen in total at most $2|\Sigma|$ times.

2. The set $T$ is updated such that $w$ causes at least one new element to be added to $T$. In this case, since the new elements added begin with characters that no other element of $T$ began with, no states will be merged and exactly one new accepting state is added to $M_{i-1}$. Noting that the maximum number of occurrences of adding new elements to $T$ is bounded by $|\Sigma|$, this can happen in total at most $|\Sigma|$ times.

3. The set $T$ is refined such that at least one element of $T$ is replaced by a proper prefix. Since the length of each element of $T$ is bounded by $\ell$, this can happen in total at most $|\Sigma| \ell$ times. 
From these observations, we have that the number of implicit errors of prediction is bounded by $\mathrm{O}(|\Sigma| \ell)$.

Noting that $|\Sigma| \leq \sum_{u \in T}(\operatorname{len}(u)+1)=\operatorname{size}(M)=n$, the following corollary follows.

COROLLARY 15 The number of implicit errors of prediction LA makes is bounded by $O(\ell n)$, where $n$ is the size of a correct SDA for the target language $L$.

Thus, from Corollary 9, Theorems 12 and 13 and Corollary 15, we have the following theorem.

THEOREM 16 The class of SDAs is polynomial-time learnable in the limit from positive data in the strict sense.

Now, suppose that we have an algorithm $\mathcal{A}$ by which a class $\mathcal{M}$ is polynomial-time learnable in the limit from positive data in the strict sense (Definition 2). And, consider a learning model in which only equivalence queries are available for learning $\mathcal{M}$. From the learnability of $\mathcal{M}$ in the strict sense, we may assume that the algorithm $\mathcal{A}$ always confines its conjectures to subsets of the target language $L(M)$ for any $M$ in $\mathcal{M}$. Then, equivalence queries can only return positive (counter)examples to $\mathcal{A}$. Further, the number of equivalence queries needed is bounded by that of implicit errors of prediction made by $\mathcal{A}$. Thus, the algorithm $\mathcal{A}$ can also achieve the polynomial-time learning of $\mathcal{M}$ using equivalence queries.

Under the same assumption, consider, in turn, a learning model in the PAC paradigm where equivalence queries can be replaced with a polynomial number of random sampling oracles $E X()$ (Angluin, 1987). Again, a polynomial number of implicit errors of prediction and the subset requirement of the algorithm $\mathcal{A}$ ensure that the number of random examples needed is bounded by a polynomial and the conjectures actually make no errors on negative data.

Thus, we have a corollary :

COROLLARY 17 The class of SDAs is polynomial-time learnable using equivalence queries only. It is also PAC-learnable in polynomial time from positive examples only.

\subsection{Incremental Feature of $L A$}

We have seen that the proposed algorithm $L A$ is a polynomial-time algorithm that learns in the limit. Note that $L A$ can actually learn a correct SDA $M$ in polynomial time in the size of $M$ and in the maximum length of data in the framework of learning in the limit. Although there are quite a few learnability results with polynomial-time efficiency, 
little is known about learning algorithms which achieve space efficiency as well. This is because it is generally hard to realize a learning strategy with restricted space, and most work proposed so far assumes unrestricted space for storing all the samples provided, in order to gain time efficiency. A learning algorithm is called iteratively consistent if and only if at each stage of learning, in order to make a new consistent guess it only uses one example and the current guess consistent with the data provided so far (Wiehagen, 1976; Jantke \& Beick, 1981; Osherson, Stob, \& Weinstein, 1986). Porat and Feldman (1991) discuss the learning problem of regular languages with finite storage. A learning algorithm with finite working storage is an algorithm that uses only finite working storage in addition to that for storing the current guess and the current example string. They showed that the class of DFAs is not learnable in the limit by any iteratively consistent algorithm with finite working storage. We note that the learning algorithm $L A$ presented in the previous section has this nice property of iterative consistency with finite additional working storage. (In fact, $L A$ can be implemented so that, besides storing the current guess and the current example, it requires at most $O(|\Sigma|)$ space for refining, parsing and merging operations.)

THEOREM 18 The class of SDAs is polynomial-time learnable in the limit from positive data by an iterative, consistent algorithm with finite working storage.

This is also in contrast to the negative result above for the whole class of DFAs.

\section{Conclusions}

In this paper, we have first proposed new definitions (Definitions 2 and 3) for polynomialtime learnability in the limit from positive data, and discussed the motivational background for introducing those concepts, by showing that any language class with an infinite descending chain property is not polynomial-time learnable in the limit from positive data in a simple definition (Definition 1) induced from Pitt's definition. Since many of the existing language classes have this property, this strongly suggested to "relax" the requirements of Definition 1 in some manner. In fact, Definition 2 has been defined as such a relaxation of Definition 1 .

Then, after introducing a subclass of DFAs called strictly deterministic automata (SDAs), we have shown that the class of SDAs is iteratively, consistently polynomialtime learnable in the limit from positive data in the strict sense, that is, in the sense of Definition 3. Note that Definition 3 requires a stronger constraint called the subset requirement than Definition 2. As is well-known, the class of DFAs is neither learnable in the limit from positive data nor polynomial-time learnable in the limit in Pitt's definition (Pitt, 1989). Hence, the main result in this paper is in marked contrast to the fact above. (Note also that, besides iterative consistency and polynomial-time efficiency, the proposed learning algorithm has additional preferable features of conservativeness and responsiveness discussed in Angluin (1980).) As a corollary, it has been shown that the 
class of SDAs is polynomial-time learnable using equivalence queries only and it is also PAC-learnable in polynomial time.

Mäkinen (1990) discusses the problem of learning Szilard languages of linear grammars compatible with a given finite sample and gives a learning algorithm for solving the problem. It is interpreted that in the "in the limit" framework his algorithm runs in linear time for updating conjectures, but no discussion of implicit prediction errors is given. The class of Szilard languages of linear grammars is a proper subclass of the class of zeroreversible languages (Angluin, 1982). Note that the class of zero-reversible languages is not polynomial-time learnable using equivalence queries only (Angluin, 1990). On the other hand, although the class of strictly regular languages is incomparable to the class of zero-reversible languages, it properly contains the class of Szilard languages of linear grammars. (In fact, a Szilard language of a linear grammar is a language accepted by an SDA, with a single final state, having $\Sigma$ as the set of labels.) Therefore, the main result in the present paper strengthens Mäkinen's result in two ways. As easily seen from the definition, the class of SDAs over an alphabet $\Sigma$ strictly depends upon the size of $\Sigma$. However, it is the case that the class is infinite even if $\Sigma$ is fixed. Further, the algorithm given in the present paper allows us to learn any SDAs with arbitrary alphabet size in time polynomial in the size of $\Sigma$, the size of a target SDA and the maximum length of all sample data. In other words, the algorithm works in polynomial time for the class of SDAs over a growing alphabet. This feature distinguishes our algorithm from most other existing ones. For future research, an interesting open problem is to find possible applications of the learnability results of SDAs to domains such as natural language acquisition (Berwick \& Pilato, 1987), and syntactic pattern recognition in general (Gonzalez \& Thomason, 1978).

\section{Acknowledgments}

The author would like to express his deepest gratitude to the referees for many useful suggestions and invaluable comments which significantly improved an early draft of the paper. In fact, many parts of this paper including Definition 2 and Theorem 1 have been contributed by the referees' efforts.

This work is supported in part by Grants-in-Aid for Scientific Research Nos. 03245104 and 04229105 from the Ministry of Education, Science and Culture, Japan.

\section{References}

Angluin, D. (1980). Inductive inference of formal languages from positive data. Information and Control, 45:117-135.

Angluin, D. (1982). Inference of reversible languages. Journal of the ACM, 29:741-765.

Angluin, D. (1987). Learning regular sets from queries and counterexamples. Information and Computation, 75:87-106.

Angluin, D. (1990). Negative results for equivalence queries. Machine Learning, 5:121-150.

Berwick, R.C. \& Pilato, S. (1987). Learning syntax by automata induction. Machine Learning, 2:9-38. 
Gold, E. M. (1967). Language identification in the limit. Information and Control, 10:447-474.

Gonzalez, R. C. \& Thomason, M. G. (1978). Syntactic Pattern Recognition: An Introduction. Addison-Wesley, Reading, Mass.

Hopcroft, J. E. \& UlIman, J. D. (1979). Introduction to Automata Theory, Languages, and Computation. Addison-Wesley, Reading, Massachusetts.

Jantke, K.P. \& Beick, H-R. (1981). Combining postulates of naturalness in inductive inference. Journal of Information Processing and Cybernetics, 17:465-484.

Mäkinen, E. (1990). The grammatical inference problem for the Szilard languages of linear grammars. Information Processing Letters, 36:203-206.

Osherson, D.N., Stob, M., \& Weinstein, S. (1986). Systems That Learn: An introduction to learning theory for cognitive and computer scientists. MIT Press, Cambridge, MA.

Porat, S. \& Feldman, J.A. (1991). Learning automata from ordered examples. Machine Learning, 7:109-138, 1991.

Pitt, L. (1989). Inductive inference, DFAs, and computational complexity. In Proceedings of 2 st Workshop on Analogical and Inductive Inference, Lecture Notes in Artificial Intelligence, Springer-Verlag 397, pp. 18-44.

Tanida, N. \& Yokomori, T. (1992). Polynomial-time identification of strictly regular languages in the limit. IEICE Transactions on Information and Systems, E75-D:125-132.

Valiant, L. (1984). A theory of the learnable. Communications of the ACM, 27:1134-1142.

Wiehagen, R. (1976). Limeserkennung rekursiver funktionen durch spezielle strategien. EIK, pp.93-99.

Wood. D. (1987). Theory of Computation. Harper and Row, New York.

Received July 16,1992

Accepted August 2, 1994

Final Manuscript August 22, 1994 